\title{
Measurements of the Total, Elastic, Inelastic and Diffractive Cross Sections with the ATLAS Detector
}

\author{
Maciej Trzebiński* ${ }^{\dagger}$ \\ on behalf of the ATLAS Collaboration \\ Institute of Nuclear Physics Polish Academy of Sciences \\ E-mail: maciej.trzebinski@ifj.edu.pl
}

The main results of the total, elastic and inelastic cross sections measurements performed by the ATLAS ALFA detectors are presented. The independent inelastic cross section measurement based on Minimum Bias Trigger Scintillators is also shown. Next, the single diffractive di-jet production and gap survival probability are briefly described. After the event selection, it was found that the diffractive component is needed for a more complete description of the data. Finally, the exclusive lepton pair production analysis in electron and muon channels is also described. The measured cross sections were found to be consistent with the theory predictions.

XXIV International Workshop on Deep-Inelastic Scattering and Related Subjects

11-15 April, 2016

DESY Hamburg, Germany

* Speaker.

$\dagger$ This work was supported in part by Polish National Science Centre under grant number UMO2015/18/M/ST2/00098 and Polish Ministry of Science and Higher Education under the Mobility Plus programme (1285/MOB/IV/2015/0). 


\section{Introduction}

Diffractive studies are one of the important parts of the physics programme for the LHC experiments. This is also true for ATLAS, where a large community works on both phenomenological and experimental aspects of diffraction. In this paper, the results of the total, elastic, inelastic and diffractive cross-sections measurements performed by the ATLAS experiment are summarised.

Diffractive processes are usually due to the exchange of a colourless object. It can be a photon (electromagnetic interactions) or a Pomeron/Reggeon (strong force). A colourless exchange may lead to one of the most prominent features of diffraction - the presence of a rapidity gap. Moreover, since such exchange does not change the quantum numbers, the interacting protons may stay intact.

\section{Total and Elastic Cross-Section Measurement}

The elastic scattering process has the simplest signature that can be imagined: two protons exchange their momentum and are scattered at small angles (see Fig. 1 (a)). Yet, the measurement is not a simple one. Firstly, as the scattering angle is relatively small, detectors must be placed far away from the Interaction Point (IP). ATLAS [1] is equipped with such devices: ALFA (Absolute Luminosity For ATLAS) detectors [2] are placed about $240 \mathrm{~m}$ from the ATLAS IP. Secondly, the LHC magnets, which are between IP and ALFA, must be specially tuned. Usually, for simplicity, magnet settings are described in terms of the betatron function at the IP, $\beta^{*}$. ALFA was designed to take data during the runs when the value of $\beta^{*}$ is large: $90 \mathrm{~m}, 1000 \mathrm{~m}$ or even $2500 \mathrm{~m}$. These settings are described in details in Ref. [3].

The measurement described in this section was done using a data sample of $80 \mu \mathrm{b}^{-1}$ collected by ATLAS at $\sqrt{s}=7 \mathrm{TeV}$. The detailed description of the analysis can be found in [4] and here only the main results are presented.

The elastic cross-section and the nuclear slope were found to be:

$$
\begin{gathered}
\sigma_{\mathrm{el}}^{\mathrm{ALFA}}(7 \mathrm{TeV})=24.00 \pm 0.19 \text { (stat.) } \pm 0.57 \text { (syst.) mb, } \\
B_{\text {nucl }}^{\mathrm{ALFA}}(7 \mathrm{TeV})=19.73 \pm 0.14 \text { (stat.) } \pm 0.26 \text { (syst.) } \mathrm{GeV}^{-2},
\end{gathered}
$$

where the first error is statistical and the second accounts for all experimental systematic uncertainties, from which the largest one is due to the luminosity uncertainty.

By using the optical theorem [5], the total cross section was determined:

$$
\sigma_{\mathrm{tot}}^{\mathrm{ALFA}}(7 \mathrm{TeV})=95.35 \pm 0.38 \text { (stat.) } \pm 1.25 \text { (exp.) } \pm 0.37 \text { (extr.) } \mathrm{mb}
$$

where the last error is related to uncertainties on the extrapolation to the zero four-momentum transfer $(|\mathrm{t}| \rightarrow 0)$.

Finally, by subtracting the elastic cross section from the total cross section, the inelastic cross section was calculated:

$$
\sigma_{\text {inel }}^{\mathrm{ALF}}(7 \mathrm{TeV})=71.34 \pm 0.36 \text { (stat.) } \pm 0.83 \text { (syst.) } \mathrm{mb} .
$$

All these results are in agreement with Monte Carlo predictions and the expected global trend [4]. 


\section{Inelastic Cross-Section Measurement}

Apart from the result described in the previous section, the inelastic cross section was independently measured by ATLAS using events triggered by Minimum Bias Trigger Scintillators (MBTS) [6, 7]. Data samples, composed of single, double, central and non-diffractive events (see Fig. 1 (b) - (e)), were collected at the centre-of-mass energies of $\sqrt{s}=7$ and $13 \mathrm{TeV}$.

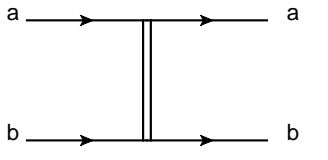

(a)

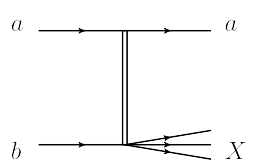

(b)

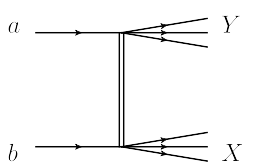

(c)

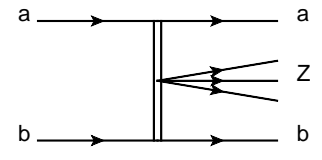

(d)

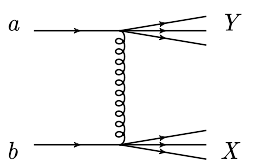

(e)

Figure 1: Diagrams of: (a) elastic scattering, (b) single, (c) double, (d) central diffraction and (e) nondiffractive scattering.

The data-taking conditions for each measurement are listed in Table 1. The minimal invariant mass is defined as the larger of the invariant masses of the two proton dissociated systems:

$$
\mathrm{M}_{\mathrm{X}}=\sqrt{s} \sum \mathrm{p}_{\mathrm{T}} e^{ \pm \eta}
$$

where $\mathrm{p}_{\mathrm{T}}$ and $\eta$ are the reconstructed transverse momentum and pseudorapidity, respectively. This quantity also defines the minimum energy lost by a proton, $\xi=\mathrm{M}_{\mathrm{X}} / s$.

Table 1: Data-taking conditions for the ATLAS inelastic cross section measurements.

\begin{tabular}{lcc}
\hline c.m. energy & $7 \mathrm{TeV}[6]$ & $13 \mathrm{TeV}[7]$ \\
integrated luminosity & $20.3 \pm 0.7 \mu \mathrm{b}^{-1}$ & $60.1 \pm 1.1 \mu \mathrm{b}^{-1}$ \\
mean number of int. per bunch crossing & 0.01 & 0.0023 \\
MBTS coverage & $2.09<|\eta|<3.84$ & $2.07<|\eta|<3.86$ \\
minimal invariant mass & $\mathrm{M}_{\mathrm{X}}>15.7 \mathrm{GeV}$ & $\mathrm{M}_{\mathrm{X}}>13 \mathrm{GeV}$ \\
visible proton energy losses & $\xi>5 \cdot 10^{-6}$ & $\xi>1 \cdot 10^{-6}$ \\
\hline
\end{tabular}

The inelastic cross sections in the fiducial regions (see Table 1) were found to be:

$$
\begin{gathered}
\sigma_{\text {fid. inel }}^{\mathrm{MBTS}}(7 \mathrm{TeV})=60.33 \pm 0.05 \text { (stat.) } \pm 0.5 \text { (syst.) } \pm 2.1 \text { (lumi.) mb } \\
\sigma_{\text {fid. inel }}^{\mathrm{MBTS}}(13 \mathrm{TeV})=68.2 \pm 0.8 \text { (exp.) } \pm 1.3 \text { (lum.) mb. }
\end{gathered}
$$

In both cases statistical uncertainty is much smaller than the systematic one. The extrapolation to the full $\xi$ range gives:

$$
\begin{gathered}
\sigma_{\text {inel }}^{\mathrm{MBTS}}(7 \mathrm{TeV})=69.4 \pm 2.4 \text { (exp.) } \pm 6.9 \text { (extr.) mb } \\
\sigma_{\text {inel }}^{\mathrm{MBTS}}(13 \mathrm{TeV})=79.3 \pm 0.8 \text { (exp.) } \pm 1.3 \text { (lum.) } \pm 2.5 \text { (extr.) mb. }
\end{gathered}
$$

As discussed in [6] and [7], the measured cross sections are in the agreement with the predictions from Monte Carlo generators: namely, Pythia6, Pythia8, Phojet at $7 \mathrm{TeV}$, and Pythia8 (various tunes), EPOS and QGSJET-II at $13 \mathrm{TeV}$. The measured cross sections agree well with theoretical predictions and are consistent with the inelastic cross section increasing with center-ofmass energy, following the trend observed at lower energies. 


\section{Single Diffractive Di-jet Measurement}

A diagram of the single diffractive di-jet production is shown in Fig. 2 (left): two jets are produced and a colour singlet (Pomeron) is exchanged from one of the interacting protons leaving it intact. Due to the fact that there can be additional (soft) interactions between the proton and final state particles, the diffractive signature can be destroyed. This effect decreases the cross-section and is quantified by a factor called the gap survival probability, $S^{2}$ [8].
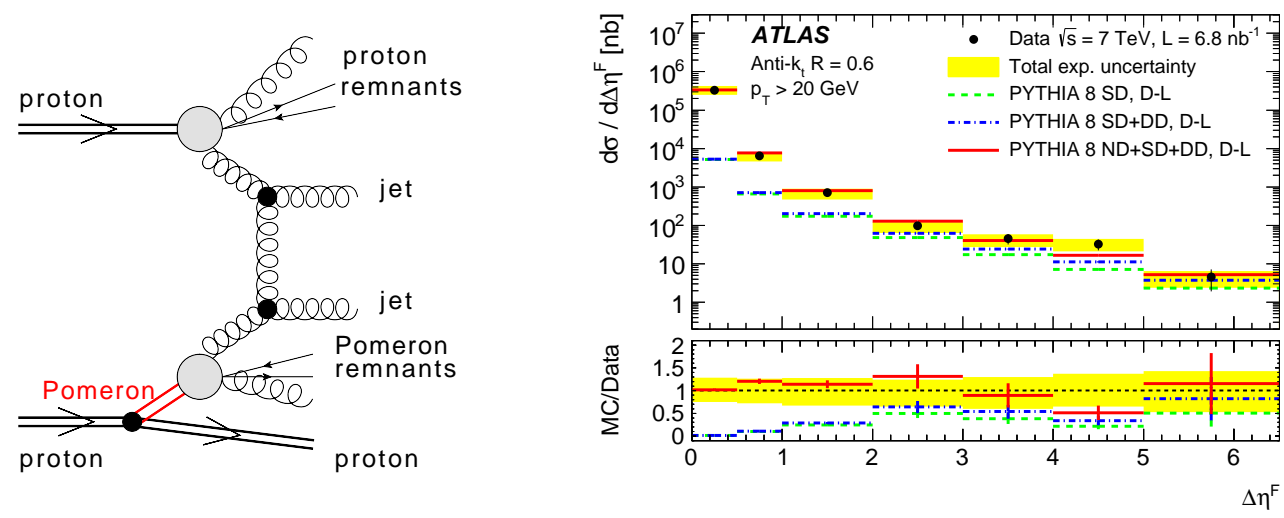

Figure 2: Left: diagram of single diffractive jet production. Right: the differential di-jet cross sections in $\Delta \eta_{F}$ compared with the PYTHIA8 predictions (from [9]).

Single diffractive di-jet production was studied using a $6.8 \mathrm{nb}^{-1}$ sample of proton-proton data collected at $\sqrt{s}=7 \mathrm{TeV}$ [9]. The average number of collisions per bunch crossing was 0.12 . The analysis was based on the selection of events containing at least two jets with $\mathrm{p}_{\mathrm{T}}>20 \mathrm{GeV}$ produced within the pseudorapidity range of $|\eta|<4$.4. Jets were reconstructed using the anti- $k_{\mathrm{T}}$ algorithm with the radius parameter, $R$, of 0.4 and 0.6 .

Events were characterised in terms of the size of the rapidity gap using a method very similar to the one described in [10]. Rapidity gaps were defined using information from trackers (charged particles with $\mathrm{p}_{\mathrm{T}}>200 \mathrm{MeV}$ and $\left.|\eta|<2.5\right)$ and calorimeters $(|\eta|<4.8$; cells with signal greater than $5 \sigma$ above the noise level). The rapidity gap, $\Delta \eta_{F}$, was defined as larger of the two empty pseudorapidity regions between the detector edge $(|\eta|=4.8)$ and the first reconstructed object.

After the event selection and comparison to the MC predictions, it was found that a diffractive component is required for a more complete description of the data, particularly when both large $\Delta \eta_{F}$ and small $\xi$ are required (see Fig. 2 (right)). Here, PYTHIA8 gave the best description of the shape and normalisation of diffractive contribution. Finally, the rapidity gap survival factor was found to be:

$$
\mathrm{S}^{2}(7 \mathrm{TeV})=0.16 \pm 0.04 \text { (stat.) } \pm 0.08 \text { (syst.). }
$$

\section{Exclusive Lepton Pair Production Measurement}

The exclusive precesses are often defined as the ones in which it is possible to measure all final state particles. In case of exclusive lepton pair production only four particles are produced: two scattered protons and two leptons (see Fig. 3 (left)). ATLAS measured such process using $4.6 \mathrm{fb}^{-1}$ of data collected at a centre-of-mass energy of $7 \mathrm{TeV}$ [11]. 

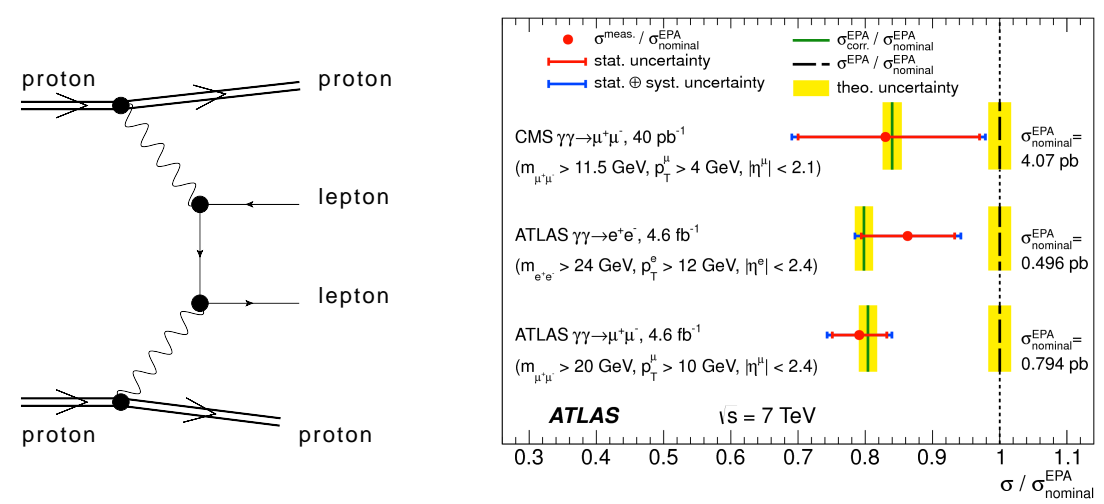

Figure 3: Left: Diagram of exclusive lepton pair production. Right: comparison of the ratios of measured (red points) and predicted (solid green lines) cross sections to the uncorrected Equivalent Photon Approximation calculations (black dashed line). From [11].

The studies were performed in electron $\left(\gamma \gamma \rightarrow e^{+} e^{-}\right)$and muon $\left(\gamma \gamma \rightarrow \mu^{+} \mu^{-}\right)$channels. In the first case, electrons and positrons were required to originate from the same vertex, have a $\mathrm{p}_{\mathrm{T}}^{e}>12 \mathrm{GeV}$ and be produced within $\left|\eta^{e}\right|<2.4$. The invariant mass of the electron system must be greater than $24 \mathrm{GeV}$. The requirements for muons were similar: the same vertex origin, $\mathrm{p}_{\mathrm{T}}{ }^{\mu}>10 \mathrm{GeV},\left|\eta^{\mu}\right|<2.4$ and $m_{\mu^{+} \mu^{-}}>20 \mathrm{GeV}$.

On top of these requirements, events had to fulfil the exclusivity criteria. Firstly, there should be no additional charged particle with $\mathrm{p}_{\mathrm{T}}>400 \mathrm{MeV}$ originating from the di-lepton vertex as well as no additional track or vertex within $3 \mathrm{~mm}$ distance from it. Secondly, in order to remove the Z-peak region, the invariant mass of the lepton pair should not lay within $70<m_{l^{+} l^{-}}<105 \mathrm{GeV}$ range. Finally, the transverse momentum of the lepton pair had to be smaller than $1.5 \mathrm{GeV}$.

In these fiducial regions, the following cross sections were obtained:

$$
\begin{aligned}
& \sigma_{\gamma \gamma \rightarrow e^{+} e^{-}}^{\text {excl. }}(7 \mathrm{TeV})=0.428 \pm 0.035 \text { (stat.) } \pm 0.018 \text { (syst.) pb } \\
& \sigma_{\gamma \gamma \rightarrow \mu^{+} \mu^{-}}^{\text {excl. }}(7 \mathrm{TeV})=0.628 \pm 0.032 \text { (stat.) } \pm 0.021 \text { (syst.) pb. }
\end{aligned}
$$

When the absorptive effects due to the finite size of the proton were taken into account in the calculation, the measured cross-sections were found to be consistent with the theory prediction ( $c f$. Fig. 3 (right)).

\section{Summary}

The ATLAS experiment was able to precisely measure the elastic and total cross sections as well as a nuclear slope at $\sqrt{s}=7 \mathrm{TeV}$ by using the forward-proton tagging technique. The inelastic cross section derived from the total and elastic components is in agreement with the independent measurement using MBTS. Moreover, all results are in accord with the MC predictions and expected global trends. In the near future, a similar precise measurements with data taken by the ATLAS/ALFA detectors at $\sqrt{s}=8$ and $13 \mathrm{TeV}$ are expected.

The measurement of single diffractive di-jet production indicates that the diffractive component is needed to describe the data. The rapidity gap survival was found to be of $S^{2}(7 \mathrm{TeV})=$ $0.16 \pm 0.04$ (stat.) \pm 0.08 (syst.). 
Finally, ATLAS has measured with a good precision the exclusive di-lepton production in electron and muon channel. Results are in agreement with theoretical predictions and that obtained by the CMS experiment [12].

It is also worth noting, that since 2016 there are new forward detectors, called ATLAS Forward Proton [13]. In future, data taken by these detectors can improve the precision of diffractive and exclusive ATLAS results $[14,15]$.

\section{References}

[1] ATLAS Collaboration, The ATLAS Experiment at the CERN Large Hadron Collider, J. Instrum. 3 (2008) S08003.

[2] ATLAS Collaboration, Technical Design Report, ATLAS-TDR-18, CERN-LHCC-2008-004.

[3] M. Trzebinski, Machine Optics Studies for the LHC Measurements, in proceedings of XXXIV-th IEEE-SPIE Joint Symposium Wilga 2014, SPIE 0277-786X, vol. 9290 (2014) 26.

[4] ATLAS Collaboration, Measurement of the total cross section from elastic scattering in pp collisions at $\sqrt{s}=7$ TeV with the ATLAS detector, Nuclear Physics B 889 (2014) 486.

[5] M. Trzebinski on behalf of the ATLAS Collaboration, Towards a Total Cross Section Measurement with the ALFA Detector at ATLAS, Acta Physica Polonica B 44 (2013) 1623.

[6] ATLAS Collaboration, Measurement of the inelastic proton-proton cross-section at $\sqrt{s}=7$ TeV with the ATLAS detector, Nature Commun. 2 (2011) 463.

[7] ATLAS Collaboration, Measurement of the inelastic proton-proton cross-section at $\sqrt{s}=13$ TeV with the ATLAS detector, ATLAS-CONF-2015-038 [arXiv:1606.02625], submitted to Phys. Rev. Lett.

[8] A. B. Kaidalov, V. A. Khoze, A. D. Martin, and M. G. Ryskin, Probabilities of rapidity gaps in high energy interactions, Eur. Phys. J. C 21 (2001) 521-529.

[9] ATLAS Collaboration, Dijet production in $\sqrt{s}=7 \mathrm{TeV}$ pp collisions with large rapidity gaps at the ATLAS experiment, Phys. Lett. B 754 (2016) 214.

[10] ATLAS Collaboration, Rapidity gap cross sections measured with the ATLAS detector in pp collisions at $\sqrt{s}=7 \mathrm{TeV}$, Eur. Phys. J. C 72 (2012) 1926.

[11] ATLAS Collaboration, Measurement of exclusive $\gamma \gamma \rightarrow l^{+} l^{-}$production in proton-proton collisions at $\sqrt{s}=7 \mathrm{TeV}$ with the ATLAS detector, Physics Letters B 749 (2015) 242.

[12] CMS Collaboration, Exclusive photon-photon production of muon pairs in proton-proton collisions at $\sqrt{s}=7 \mathrm{TeV}$, JHEP 1201 (2012) 052,

[13] ATLAS Collaboration, Technical Design Report for the ATLAS Forward Proton Detector, CERN-LHCC-2015-009, ATLAS-TDR-024.

[14] ATLAS Collaboration, Exclusive Jet Production with Forward Proton Tagging, ATL-PHYS-PUB-2015-003,

M. Trzebinski, R. Staszewski, J. Chwastowski, On the Possibility of Measuring the Single-tagged Exclusive Jets at the LHC, Eur. Phys. J. C 75 (2015) 320,

M. Trzebinski, Exclusive Jet Measurement in Special LHC Runs - Feasibility Studies, to be published in proceedings of XXII Cracow Epiphany Conference.

[15] N. Cartiglia (ed.), C. Royon (ed.) et al., LHC Forward Physics, CERN-PH-LPCC-2015-001, SLAC-PUB-16364, DESY 15-167. 